\title{
Megaloblastic Anemia: A Drug-Induced Disorder
}

\section{Satadal Barik ${ }^{*}$}

Department of Microbiology, Kumar Bhaskar Varma Sanskrit \& Ancient Studies University, Nalbari, Assam, India

*Corresponding author: Satadal Barik, Department of Microbiology, Kumar Bhaskar Varma Sanskrit \& Ancient Studies University, Nalbari, Assam, India, Tel: 03624298 311; E-mail: satadalbarik1@gmail.com

Received date: Jun 02, 2016, Accepted date: Jun 08, 2016, Publication date: Jun 13, 2016

Copyright: (c) 2016 Barik S. This is an open-access article distributed under the terms of the Creative Commons Attribution License, which permits unrestricted use, distribution, and reproduction in any medium, provided the original author and source are credited.

Megaloblastic anemia is categorized by distinguished hematopoietic cell morphology, unproductive hematopoiesis, induced by folic acid and/or vitamin $\mathrm{B}_{12}$ deficiency [1]. Ineffective DNA synthesis in hematopoietic progenitor cells or myelodysplastic syndrome leading to congenital disorders is commonly responsible for the megaloblastic anemia. In western populations, cobalamin (vitamin $\mathrm{B}_{12}$ ) deficiency is a common factor to cause the megaloblastic anemia. The complexity of the clinical manifestations of megaloblastic anemia often leads to a misdiagnosis in the geriatric population. Bone marrow aspirate examination and bone marrow core biopsy can aid in the morphologic diagnosis of this disorder. The diagnosis of megaloblastic anemia is quite excellent and uptake of folic acid and/or vitamin $\mathrm{B}_{12}$ with a simple regimen is effective for the treatment [2]. In a case report, a 31year old army soldier with both vitamin $\mathrm{B}_{12}$ and folic acid deficiency in his blood, showed a typical megaloblastic anaemia with oval macrocytes and hypersegmented neutrophils. Three months later, the patient revealed a further reduction in his neutrophil count and a mild thrombocytopaenia. The repeat bone marrow examination exposed a considerable (33\%) plasma cell penetratation with erythroid dysplasia and further investigations confirmed a non secretory multiple myeloma (NSMM). The initial presentation was megaloblastic anaemia but the patient was diagnosed with NSMM later [3].

Another report concerning a 75 year old male teacher who experienced dizziness, nausea, vomiting and bilateral weakness in both lower extremities was diagnosed as megaloblastic anemia and successfully treated with the routine course of treatment included folic acid and vitamin $B_{12}$. Routine blood examination revealed a significant increment of lactate dehydrogenase, ineffective hematopoiesis in bone marrow and a mild jaundice was detected. Furthermore bone marrow examination supported the diagnosis of megaloblastic anemia. The initial course of treatment with folic acid and vitamin $B_{12}$ significantly improved the bone marrow morphology, alleviated dizziness and fatigue, as did the hallucinations [4].

In a perspective piece of review article, Hesdorffer and Longo [5] analyzed the drug-induced megaloblastic anemia, unfolding biochemical processes and gave importance to the most familiar drugs involved in this disorder [5]. Though most common drugs involved in megaloblastic anemia were included in this study, there are few more drugs could be the centre of attention which are related to this disease. I discuss briefly about these drugs and the inclusion of them could influence the outcome of this study. Sunitinib, a multitargeted tyrosine kinase inhibitor can also incite macrocytosis and consequent anemia. An association between sunitinib-based treatment and macrocytosis was established earlier. In a cumulative study, 43 patients had been treated with sunitinib; among them $49 \%$ patients developed macrocytosis and $86 \%$ patients developed anemia during the treatment
[6]. Treatment with sunitinib may increase the mean corpuscular volume and suggested the hypothesis that this increase is related to the metabolism of vitamin $B_{12}$ or folate. An investigation can be cited in order to define this phenomenon. Six patients were treated with sunitinib; an increment of mean corpuscular volume was visualised in two patients before a drop in cobalamin levels [7].

These data suggested that cobalamin deficiency could be involved in sunitinib-induced macrocytosis. Triamterene, a potassium-sparing diuretic, causes functional folic acid deficiency by inhibiting dihydrofolate reductase which obstructs the metabolism of folic acid, thus leading to dangerous megaloblastic anemia. Patients with a borderline folate deficiency should use this drug with caution [8]. Antiseizure medicines, such as primidone and valproic acid, may play a crucial role in enhancing folate metabolism and enzyme induction, thus inducing megaloblastic anemia. Zidovudine is a thymidine analogue that acts as a nucleoside reverse-transcriptase inhibitor, can cause the impairment of DNA synthesis that leading to a macrocytosis with megaloblastic erythropoiesis [9]. Orlistat, a specific inhibitor of gastrointestinal lipases that may delay the growth of diabetes mellitus, have the adverse effects to cause thrombopenia and macrocytic anemia [10].

There are few more drugs including pyrazinamide, an antitubercular drug, as well as acyclovir, lithium and phenylbutazone can also be involved to cause megaloblastic anemia. The inclusion of these drugs may change the multivariate analysis and ultimately the conclusion. Additional studies are necessary on the mechanism of drug-induced macrocytosis as well as the megaloblastic anemia.

\section{References}

1. Clarke R, Grimley Evans J, Schneede J, Nexo E, Bates C, et al. (2004) Vitamin B12 and folate deficiency in later life. Age Ageing 33: 34-41.

2. Ide E, Van Biervliet S, Thijs J, Vande Velde S, De Bruyne R, et al. (2011) Solid food refusal as the presenting sign of vitamin B12 deficiency in a breastfed infant. Eur J Pediatr 170: 1453-1455.

3. Priyankara WD, Gunawardena D (2013) Megaloblastic anaemia in non secretory multiple myeloma: a rare presentation. Ceylon Med J 58: 89-90.

4. Zhang Q, Lv XY, Yang YM (2015) Megaloblastic anemia with hypotension and transient delirium as the primary symptoms: report of a case. Int J Clin Exp Med 8: 18879-18883.

5. Hesdorffer CS, Longo DL (2015) Drug-Induced Megaloblastic Anemia. N Engl J Med 373: 1649-1658.

6. Price J, Shaarbaf R, Wood L (2010) Sunitinib causes macrocytosis in patients with advanced renal cell carcinoma. Curr Oncol 17: 30-33.

7. Gillessen S, Graf L, Korte W, Cerny T (2007) Macrocytosis and cobalamin deficiency in patients treated with sunitinib. N Engl J Med 356: 2330-2331.

8. Lieberman FL, Bateman JR (1968) Megaloblastic anemia possibly induced by triamterene in patients with alcoholic cirrhosis. Two case reports. Ann Intern Med 68: 168-173. 
Citation: Barik S (2016) Megaloblastic Anemia: A Drug-Induced Disorder. J Blood Disord Transfus 7: 359. doi:10.4172/2155-9864.1000359

Page 2 of 2

9. Wickramasinghe SN (2006) Diagnosis of megaloblastic anaemias. Blood Rev 20: 299-318.
10. Palacios-Martinez D, Garcia-Alvarez JC, Montero-Santamaria N, VillarRuiz OP, Ruiz-Garcia A, et al. (2013) Macrocytic anemia and thrombocytopenia induced by orlistat. Int J Endocrinol Metab 11: e6721. 